\title{
3 Research Square

\section{Mid-term results of an anterior ilioischial plating for complex acetabular fractures with a combined anterior approach: a retrospective cohort study}

\section{Weikun Meng}

Trauma Center, Second People's Hospital of Yunnan

\section{Yi Ou}

Trauma Center, Second People's Hospital of Yunnan

\section{Zhaoxiang Wu}

Trauma Center, Second People's Hospital of Yunnan

\section{Hongchang Yang}

Trauma Center, Second People's Hospital of Yunnan

\section{Ge Chen}

Trauma Center, Second People's Hospital of Yunnan

\section{Xin Bi}

Trauma Center, Second People's Hospital of Yunnan

\section{Liang Gao ( $\sim$ lianggao22@gmail.com )}

Center of Experimental Orthopaedics, Saarland University https://orcid.org/0000-0003-1438-9670

\section{Zhong Chen}

Trauma Center, Second People's Hospital of Yun

\section{Research article}

Keywords: Complex acetabular fracture, Anterior plating, Ilioischial plate, Modified Stoppa approach, Ilioinguinal approach

Posted Date: June 17th, 2019

DOl: https://doi.org/10.21203/rs.2.10376/v1

License: (c) (i) This work is licensed under a Creative Commons Attribution 4.0 International License. Read Full License 


\section{Abstract}

Background: Bi-columnar plating via combined anterior and posterior approaches is routinely applied treatment for complex acetabular fractures with noteworthy shortcomings. The goal of this study was to evaluate the effectiveness of the anterior plating with an ilioischial plate through the combined anterior approach for complex acetabular fracture treatment. Methods: Patients with complex acetabular fractures treated at our trauma centre between January 2015 and December 2016 were retrospectively reviewed. Fractures were stabilized with an ilioischial plate via a modified Stoppa approach combined with the 1st window of the ilioinguinal approach. Interval between injury and surgery, operative time and intraoperative blood loss were recorded. Fracture reduction was evaluated radiographically with the modified Matta's criteria at 3 days postoperatively. Postoperative hip function was assessed using the modified Merle d'Aubigne and Postel clinical grading system over at least 1 year postoperatively. Results: Twenty patients were included with an average follow-up of 17 months (range, 13-28). The mean time interval between injury and surgery was 7.0 days (range, 3-13 days), operative time was 2.0 hours (range, 1.4-3.2 hours), and intraoperative blood loss was $320 \mathrm{~mL}$ (range, $220-450 \mathrm{~mL}$ ). Fracture reduction was excellent in 15 cases (75\%), good in 3 cases (15\%), fair in 1 case (5\%), and poor in 1 case (5\%). Final hip function was excellent in 13 cases (68\%), good in 3 cases (16\%), fair in 2 cases (11\%), and poor in 1 case (5\%). Bony healing was achieved in all cases and few complications were reported including recoverable obturator nerve injuries in 2 patients (10\%) and traumatic arthritis in 1 patient (5\%). Conclusions: Anterior fixation of complex acetabular fractures with an ilioischial plate via the combined anterior approach yielded clinically satisfactory mid-term outcomes, providing a potentially effective alternative to the conventional treatment algorithm.

\section{Background}

Complex acetabular fractures involving both anterior and posterior columns are majorly caused by highenergy injuries [1, 2]. Their postoperative outcomes are influenced by several factors, including noncontrollable (type of fracture/dislocation, damage to femoral head, associated injuries and co-morbidity) or controllable (timing of surgery, surgical approach, quality of reduction and complications) [3]. Surgical intervention is aimed to use the least invasive approach that achieves anatomic reduction of the articular surface and stable fixation while minimizing the risks of early and late complications [4].

Controversies still exist regarding the optimal surgical approach for achieving better exposure of fracture sites, less soft tissue damage and lower complications rates. Simultaneous anterior and posterior approaches are routinely used to fix complex acetabular fractures with both anterior and posterior plating, which have noteworthy drawbacks (e.g. long operating time and substantial blood loss) and complications (e.g. structure damage, infection, abductor weakness, hernia) [5-11]. The modified Stoppa approach, an anterior intrapelvic approach initially used for inguinal hernia surgery, can approach the anterior acetabulum and pelvic bone. The ilioinguinal approach, another anterior approach, allows exposure to entire internal iliac fossa and pelvic brim from the sacroiliac joint to the pubic symphysis, quadrilateral surface of innominate bone and superior/inferior pubic rami (thus allows exposure of 
anterior column), and portion of external aspect of ilium. For specific complex acetabular fractures, combining the modified Stoppa approach with the 1st window of the ilioinguinal approach [12] was previously reported in $60 \%$ [13] to $93 \%$ [14] cases, and others [15-17] described this combination as a standard procedure. However, few studies have ever performed to investigate such a combined approach with secured anterior plate fixation as a potentially effective alternative to the conventional Bi-columnar plating via combined anterior and posterior approach.

This study aimed to evaluate the mid-term clinical outcomes of with solely anterior plating with the modified Stoppa approach combined with the 1st window of the ilioinguinal approach to treat complex acetabular fractures.

\section{Methods}

\section{Patients}

Patients with complex acetabular fractures operated at our trauma centre by the identical senior surgeon team between January 2015 and December 2016 were retrospectively reviewed with the approval of local ethics committee. Patients were included with the following criteria: (1) acute traumatic acetabular fracture and (2) one of the following fracture types according to the Letournel classification [18]: both columns, T-shaped fractures, transverse fracture, anterior column/wall and posterior hemitransverse fractures. Patients with (1) missing data, (2) osteoporosis or osteoarthritis, and (3) systemic illnesses (e.g. hyperthyroidism, hyperparathyroidism, androgen deficiency, malabsorption, and neoplasia) were excluded. All patients underwent a standardized assessment, including history taking, clinical examination and imaging investigations. Fracture type and displacement was assessed with plain radiographs and computed tomography (CT) with 3D reconstruction.

\section{Surgical procedure}

Patients were placed in a supine position allowing for unrestricted movements of the lower limbs. The affected lower limb was sterilized and wrapped to the middle of the thigh. The hip and knee of the affected limb were flexed slightly to relax the iliopsoas and neurovascular bundle. A combined approach of the modified Stoppa approach and the 1st window of the ilioinguinal approach was undertaken as previously described $[12,19]$. A Schanz screw was inserted into the proximal femur allowing for intraoperative manual traction and fractures were reduced with assistance of different reduction instruments (e.g. ball spike and reduction forceps). Fragments were temporarily fixated with Kirschner wires and anatomic reconstruction of the different fracture fragments was confirmed. Infrapectineal or suprapectineal plates ( $3.5 \mathrm{~mm}$; 8 hole) contoured along the pelvic brim was anchored with cortical screws above the sciatic notch. Another plate, termed as the "ilioischial plate", was contoured over the pelvic brim to buttress comminution or counteract posterior column medial displacement (Figure 1). Displaced quadrilateral surface fragments were meticulously reduced and buttressed with the bridging effect of the 
ilioischial plate. Anchorage of the ilioischial plate was attained with cortical screws to the iliac fossa and the safe zone between the sciatic buttress and the ischial spine. For specific cases with comminuted fragments of the posterior column, an additional lag screw was inserted from anterior to posterior direction within the safe zone to fix those fragments to provide further stabilization of the posterior fragments. Operative data were recorded for each patient including interval between injury and surgery, operative time and intraoperative blood loss.

\section{Postoperative management}

The operatized limb was elevated slightly with both hip and knee appropriately flexed. Wound drainage was removed 24-72 hours postoperatively. Oral administration of celecoxib was applied for pain control and heterotopic ossification prevention. Low-molecular-weight heparin was used for thrombosis prevention. Rehabilitation was initiated after 24 hours postoperatively to improve blood circulation and prevent deep vein thrombosis and muscular atrophy. Ambulation with crutches was allowed at 3 days postoperatively. Partial weight-bearing exercises and ambulation without crutches were started at the postoperative 6 and 12 weeks, respectively.

\section{Outcome evaluation}

Quality of the fracture reduction was evaluated with the modified Matta's criteria on the plain radiographs at 3 days postoperatively [20]. Reduction was classified as: excellent (anatomical reduction), good (0-1 $\mathrm{mm}$ residual displacement), fair (2-3 $\mathrm{mm}$ residual displacement), and poor ( $>3 \mathrm{~mm}$ residual displacement). Function of the affected hip was evaluated using the modified Merle d'Aubigne and Postel scoring system over one year postoperatively [21]. Hip function was considered to be: excellent (no pain, a normal gait, a range of joint motion $>75 \%$, no sign of arthritic changes or mild joint space narrowing or sclerosis on X-ray), good (mild pain, a normal gait, a range of joint motion $>50 \%$, joint surface sclerosis or joint space narrowing or osteophyte formation), fair (moderate pain, mild abnormality of gait, a range of joint motion $<50 \%$, significant joint space narrowing or joint surface sclerosis and osteophyte formation), and poor (severe pain, obvious gait abnormality, joint stiffness with deformity, significant arthritic changes or obvious dislocation of the femoral head).

\section{Statistical analysis}

Descriptive statistics are used to describe the results. Data are presented as the mean \pm standard deviation, range, and percentage, where appropriate.

\section{Results}

\section{Demographic characteristics}


A total of 20 patients were enrolled in this study, including 12 males (60\%) and 8 females (40\%), aged 16 to 62 (mean 45) years old. Nine cases (45\%) were left-sided fractures and 11 cases (55\%) were right-sided fractures. According to the Letournel classification, 2 cases (10\%) were classified as both columns fracture, 6 cases (30\%) as anterior with posterior hemi transverse fracture, 10 cases $(50 \%)$ as T-shaped fracture, and 2 cases (10\%) as transverse fracture [18]. The postoperative follow-up was over 12 months (mean 17, range, 13-28 months), and only one patient was lost after 3 months' follow-up (Table 1).

\section{Operative characteristics}

The mean time interval between injury and surgery was 7.0 days (range, 3-13 days), operative time was 2.0 hours (range, 1.4-3.2 hours), and intraoperative blood loss was $320 \mathrm{~mL}$ (range, 220-450 mL).

\section{Radiographic outcomes}

Bone healing was achieved in all 19 cases followed up over 3 months. Based on Matta's criteria, postoperative radiographic outcome was excellent in 15 cases (75\%) (Figure 2, Figure 3), good in 3 cases (15\%), fair in 1 case (5\%) and poor in 1 case (5\%) (Table 2).

\section{Clinical outcomes}

According to the modified Merle d'Aubigne and Postel scoring system, the function of the affected hip joint was excellent in 13 cases (68\%), good in 3 cases (16\%), fair in 2 cases (11\%), and poor in 1 case (5\%) (Table 2).

Two patients (10\%) exhibited clinical evidences of obturator nerve injuries, which were recovered fully after 3 months without special medical interventions. One patient (5\%) developed symptomatic osteoarthritis 2 years after surgery, which was diminished by medications. No other specific complications were reported, including internal fixation failure, postoperative infection, and heterotopic ossification.

\section{Discussion}

This retrospective study showed clinically satisfactory mid-term outcomes of the anterior ilioischial plating for complex acetabular fractures via the modified Stoppa approach combined with the $1 \mathrm{st}$ window of the ilioinguinal approach. Such a simplified approach with solely anterior plating might provide an effective alternative to the conventional simultaneous anterior and posterior approaches with potential clinical advantages.

The main findings of this study were the clinically satisfactory outcomes of complex acetabular fractures treated with solely anterior plating via the combined anterior approach at an average 17-month follow-up. 
Such a combined anterior approach, previously observed in 60\% [13] to 93\% [14] cases, was proposed as a standard procedure for specific complex acetabular fractures [15-17]. Bone healing was achieved in all our cases at 3 months postoperatively. Good or excellent fracture abduction and fixation was observed in $90 \%$ cases, which was in good agreement with a previous study (73 complex fractures) from Kumar and colleagues reporting congruent reduction in $89 \%$ cases treated with either single (anterior or posterior) approach or simultaneous anterior-posterior approach [22]. Moreover, $85 \%$ of our cases achieved good or excellent hip function at average 17-month postoperatively, which is consistent with the prior investigation (18 complex fractures) reporting $85.2 \%$ cases with good or excellent hip function over 4 years follow-up after treatment by simultaneous ilioinguinal and Kocher-Langenbeck approaches [23].

Additionally, the afore-mentioned combined anterior approach is practically convenient to access fracture fragments of posterior column effectively with advantages of less blood loss, shorter operation time, and fewer complications than traditional approaches. The average intraoperative blood loss of our cohorts was $320 \mathrm{~mL}$, which was significantly less than blood loss with a single ilioinguinal approach ranging from $760 \mathrm{~mL}$ to 1170 [24-26] or simultaneous anterior (modified ilioinguinal/Stoppa approach) and Kocher-Langenbeck approach ranging from $586 \mathrm{ml}$ to $1252 \mathrm{ml}$ [26-30]. The average operative time was 2.1 hours in our cohorts, compared with 2.6-4.3 hours for a single ilioinguinal approach $[24,25]$ and 2.14.4 hours for the simultaneous anterior (modified ilioinguinal/Stoppa approach) and Kocher-Langenbeck approach [27-29, 31, 32]. Moreover, no heterotopic ossification and only one obturator nerve injury (recovered within 3 months postop) was observed in our cohorts, which are significantly less than the rates of heterotopic ossification (25.6\%), traumatic nerve palsy (16.4\%), and iatrogenic nerve palsy ( $8.0 \%)$ reported by a recent meta-analysis [3].

Previous biomechanical experiment demonstrated that a single locking plating of the anterior column provided less stability than a traditional treatment with posterior column plating and anterior column screwing [33]. In practice, the anterior fixation with multiple spring plates, especially the ilioischial plate, allows for a direct buttressing of posterior column fractures and provides clinically comparable stability as a posterior plating. Additional lag screws can be applied from anterior to posterior direction to fix the fragments of the posterior columns and achieve a rigid fixation [34, 35].

Surgical indications of such a simplified approach are proposed as follows: (1) a posterior column fracture that can be straightforwardly reduced, (2) a T-shaped or transverse fracture without posterior wall fracture, (3) an anterior column fracture with a concomitant transverse posterior column fracture, and (4) fractures with few posterior fracture fragments and minimal involvement of the weight bearing area. Preoperative evaluation of patient status, fracture type, extent of the displacement, the surgeon's experience is crucial [32]. Other approaches might be chosen for patients with obesity or history of previous low abdominal surgeries (causing local adhesions). Moreover, imprudent manipulation with limited exposure might cause extensive soft tissue damages and hinder the satisfactorily reduction of fractures, which possibly influences the long-term prognosis [36-38]. Therefore, surgeons are recommended to utilize the Stoppa approach after mastering the ilioinguinal approach to avoid iatrogenic structure injuries of the perifracture site. 
Several limitations exist in this study. The sample size was relatively small, and no biomechanical experiments were performed to quantitatively validate the stability of the ilioischial plating. Secondly, the retrospective analysis cannot allow for a detailed investigation of characteristics of our cohorts which might alter the ultimate outcomes. Moreover, long-term comparisons with traditional treatments are required to confirm the possible benefits of this simplified surgical modality.

\section{Conclusions}

Anterior fixation of complex acetabular fractures with an ilioischial plate through the modified Stoppa approach combined with the 1 st window of the ilioinguinal approach yielded clinically satisfactory midterm outcomes. Such a simplified surgical modality provides a valuable venue for complex acetabular fractures treatment with clinical advantages over the conventional treatment algorithm.

\section{Trial Registration Information}

The trial was retrospectively registered in https://www.researchregistry.com (No. Researchregistry4862) on May 12, 2019. The first participant was enrolled on January 13, 2015.

\section{Abbreviations}

3D: Three-dimensional; CT: Computed Tomography.

\section{Declarations}

\section{Funding}

The authors received no specific individual funding for this work.

\section{Availability of data and materials}

The data supporting your findings can be found and have be presented within the manuscript.

\section{Authors' contributions}

ZC conceived and designed the study. WKM, YO, ZXW, GC, XB, HCY, and LG performed the data analysis. WKM, YO and LG drafted the initial manuscript with all authors contributing substantially to the final manuscript. All authors read and approved the final manuscript.

\section{Ethics approval and consent to participate}


Ethical approval was obtained from the Institutional Review Board of the Second People's Hospital of Yunnan Province, Kunming/Yunnan, P. R. China.

\section{Consent for publication}

Informed consent was obtained from all individual participants included in the study.

\section{Competing interests}

The authors declare that they have no competing interests.

\section{References}

1. Sagi HC, Jordan CJ, Barei DP, Serrano-Riera R, Steverson B: Indomethacin prophylaxis for heterotopic ossification after acetabular fracture surgery increases the risk for nonunion of the posterior wall. Journal of orthopaedic trauma 2014, 28(7):377-383.

2. Rickman M, Young J, Trompeter A, Pearce R, Hamilton M: Managing acetabular fractures in the elderly with fixation and primary arthroplasty: aiming for early weightbearing. Clinical orthopaedics and related research 2014, 472(11):3375-3382.

3. Giannoudis PV, Grotz MR, Papakostidis C, Dinopoulos H: Operative treatment of displaced fractures of the acetabulum. A meta-analysis. The Journal of bone and joint surgery British volume 2005, 87(1):2-9.

4. Fractures of the Pelvis and Acetabulum. In: Principles and Methods of Management. edn. Edited by M T, DL H, JF K, M V. Stuttgart: Thieme; 2015.

5. Routt ML, Jr., Swiontkowski MF: Operative treatment of complex acetabular fractures. Combined anterior and posterior exposures during the same procedure. The Journal of bone and joint surgery American volume 1990, 72(6):897-904.

6. Harris AM, Althausen P, Kellam JF, Bosse MJ: Simultaneous anterior and posterior approaches for complex acetabular fractures. Journal of orthopaedic trauma 2008, 22(7):494-497.

7. Assal M, Ray A, Fasel JH, Stern R: A modified posteromedial approach combined with extensile anterior for the treatment of complex tibial pilon fractures (AO/OTA 43-C). Journal of orthopaedic trauma 2014, 28(6):e138-145.

8. Matta JM: Operative treatment of acetabular fractures through the ilioinguinal approach. A 10-year perspective. Clinical orthopaedics and related research 1994(305):10-19. 
9. Kloen P, Siebenrock KA, Ganz R: Modification of the ilioinguinal approach. Journal of orthopaedic trauma 2002, 16(8):586-593.

10. Helfet DL, Schmeling GJ: Management of complex acetabular fractures through single nonextensile exposures. Clinical orthopaedics and related research 1994(305):58-68.

11. Rommens PM, Broos PL, Vanderschot P: [Preparation and technique for surgical treatment of 225 acetabulum fractures. 2 year results of 175 cases]. Der Unfallchirurg 1997, 100(5):338-348.

12. Judet R, Judet J, Letournel EJJ: Fractures of the acetabulum: classification and surgical approaches for open reduction: preliminary report. 1964, 46(8):1615-1675.

13. Sagi HC, Afsari A, Dziadosz DJJoot: The anterior intra-pelvic (modified rives-stoppa) approach for fixation of acetabular fractures. 2010, 24(5):263-270.

14. Khoury A, Weill Y, Mosheiff RJOOuT: The Stoppa approach for acetabular fracture. 2012, 24(4-5):439448.

15. Andersen RC, O'Toole RV, Nascone JW, Sciadini MF, Frisch HM, Turen CWJJoot: Modified stoppa approach for acetabular fractures with anterior and posterior column displacement: quantification of radiographic reduction and analysis of interobserver variability. 2010, 24(5):271-278.

16. Jakob M, Droeser R, Zobrist R, Messmer P, Regazzoni PJJoT, Surgery AC: A less invasive anterior intrapelvic approach for the treatment of acetabular fractures and pelvic ring injuries. 2006, 60(6):13641370.

17. Laflamme G, Hebert-Davies J, Rouleau D, Benoit B, Leduc SJI: Internal fixation of osteopenic acetabular fractures involving the quadrilateral plate. 2011, 42(10):1130-1134.

18. Beaulé PE, Dorey FJ, Matta JMJJ: Letournel classification for acetabular fractures: assessment of interobserver and intraobserver reliability. 2003, 85(9):1704-1709.

19. Isaacson MJ, Taylor BC, French BG, Poka AJCO, Research ${ }^{\circledR}$ R: Treatment of acetabulum fractures through the modified Stoppa approach: strategies and outcomes. 2014, 472(11):3345-3352.

20. Matta JM: Fractures of the acetabulum: accuracy of reduction and clinical results in patients managed operatively within three weeks after the injury. The Journal of bone and joint surgery American volume 1996, 78(11):1632-1645.

21. d'Aubigne RM, Postel M: The classic: functional results of hip arthroplasty with acrylic prosthesis. 1954. Clinical orthopaedics and related research 2009, 467(1):7-27.

22. Kumar A, Shah N, Kershaw S, Clayson AJl: Operative management of acetabular fractures: a review of 73 fractures. 2005, 36(5):605-612. 
23. Moroni A, Caja V, Sabato C, Zinghi GJl: Surgical treatment of both-column fractures by staged combined ilioinguinal and Kocher-Langenbeck approaches. 1995, 26(4):219-224.

24. Bi DW, Han L, Zu G, Zheng Q, Fei J, Wei W, Gu J, Ma HT, Chen YM, Hu GF: [Choice of operative approaches for complex fractures of the quadrilateral area of acetabular]. Zhongguo gu shang = China journal of orthopaedics and traumatology 2013, 26(11):944-948.

25. Rocca G, Spina M, Mazzi M: Anterior Combined Endopelvic (ACE) approach for the treatment of acetabular and pelvic ring fractures: A new proposal. Injury 2014, 45 Suppl 6:S9-S15.

26. Elmadag M, Guzel Y, Acar MA, Uzer G, Arazi M: The Stoppa approach versus the ilioinguinal approach for anterior acetabular fractures: a case control study assessing blood loss complications and function outcomes. Orthopaedics \& traumatology, surgery \& research : OTSR 2014, 100(6):675-680.

27. Fan L, Jin YJ, He L, Lu Z, Fan HH: [Modified Stoppa approach in treatment of pelvic and acetabular fractures]. Zhongguo gu shang = China journal of orthopaedics and traumatology 2012, 25(10):810-812.

28. Sagi HC, Afsari A, Dziadosz D: The anterior intra-pelvic (modified rives-stoppa) approach for fixation of acetabular fractures. Journal of orthopaedic trauma 2010, 24(5):263-270.

29. Sharma AR, Jagga S, Lee SS, Nam JS: Interplay between Cartilage and Subchondral Bone Contributing to Pathogenesis of Osteoarthritis. International Journal of Molecular Sciences 2013, 14(10):19805-19830.

30. Elmadag M, Guzel Y, Aksoy Y, Arazi M: Surgical Treatment of Displaced Acetabular Fractures Using a Modified Stoppa Approach. Orthopedics 2016, 39(2):e340-345.

31. Wang P, Zhu X, Xu P, Zhang Y, Wang L, Liu X, Mu W: Modified ilioinguinal approach in combined surgical exposures for displaced acetabular fractures involving two columns. SpringerPlus 2016, $5(1): 1602$.

32. Kim HY, Yang DS, Park CK, Choy WS: Modified Stoppa approach for surgical treatment of acetabular fracture. Clinics in orthopedic surgery 2015, 7(1):29-38.

33. Khajavi K, Lee AT, Lindsey DP, Leucht P, Bellino MJ, Giori NJ: Single column locking plate fixation is inadequate in two column acetabular fractures. A biomechanical analysis. Journal of orthopaedic surgery and research 2010, 5:30.

34. Mehin R, Jones B, Zhu Q, Broekhuyse HJCJoS: A biomechanical study of conventional acetabular internal fracture fixation versus locking plate fixation. 2009, 52(3):221.

35. Marintschev I, Gras F, Schwarz CE, Pohlemann T, Hofmann GO, Culemann UJI: Biomechanical comparison of different acetabular plate systems and constructs-the role of an infra-acetabular screw placement and use of locking plates. 2012, 43(4):470-474. 
36. Mears DCJJ-JotAAoOS: Surgical treatment of acetabular fractures in elderly patients with osteoporotic bone. 1999, 7(2):128-141.

37. Jeffcoat DM, Carroll EA, Huber FG, Goldman AT, Miller AN, Lorich DG, Helfet DLJJoot: Operative treatment of acetabular fractures in an older population through a limited ilioinguinal approach. 2012, 26(5):284-289.

38. Routt JM, Swiontkowski MJTJob, volume jsA: Operative treatment of complex acetabular fractures. Combined anterior and posterior exposures during the same procedure. 1990, 72(6):897-904.

\section{Tables}

Table 1. Demographic and surgery characteristics.

\begin{tabular}{lcc}
\hline Variables & Patients & Range/ Percentage (\%) \\
& $(\mathrm{n}=20)$ & \\
\hline Age (years) & 45 & $16-62$ \\
Gender & & \\
\hline Female & 8 & 60 \\
\hline Male & 12 & 40 \\
\hline Side of injury & 11 & 55 \\
\hline Right & 9 & 45 \\
\hline Left & 2 & 10 \\
\hline Letournel classification & 6 & 30 \\
\hline Both columns fracture & 10 & 50 \\
\hline Anterior with posterior hemitransverse fracture & 2 & 10 \\
\hline T-shaped fracture & 7.0 & $3-13$ \\
\hline Transverse fracture & 2.0 & $1.4-3.2$ \\
\hline Time from injury-to-surgery (days) & 320 & $220-450$ \\
\hline Operation time (hours) & & \\
\hline Intraoperative blood loss (ml) & & \\
\hline
\end{tabular}


Table 2. Assessments of postoperative radiographic and clinical outcomes.

\begin{tabular}{lcc}
\hline Outcome evaluations $^{*}$ & Results & Percentage (\%) \\
\hline Matta's criteria* $^{\text {Excellent }}$ & & \\
Good & 15 & 75 \\
\hline Fair & 3 & 15 \\
\hline Poor & 1 & 5 \\
\hline Modified Merle d' Aubigne \& Postel scoring system ${ }^{\dagger}$ & 1 & 5 \\
$\quad$ Excellent & 13 & \\
\hline Good & 3 & 68 \\
\hline Fair & 2 & 16 \\
Poor & 1 & 5 \\
\hline
\end{tabular}

*at 3 days postoperatively; ${ }^{\dagger}$ at over 1 year postoperatively.

\section{Figures}
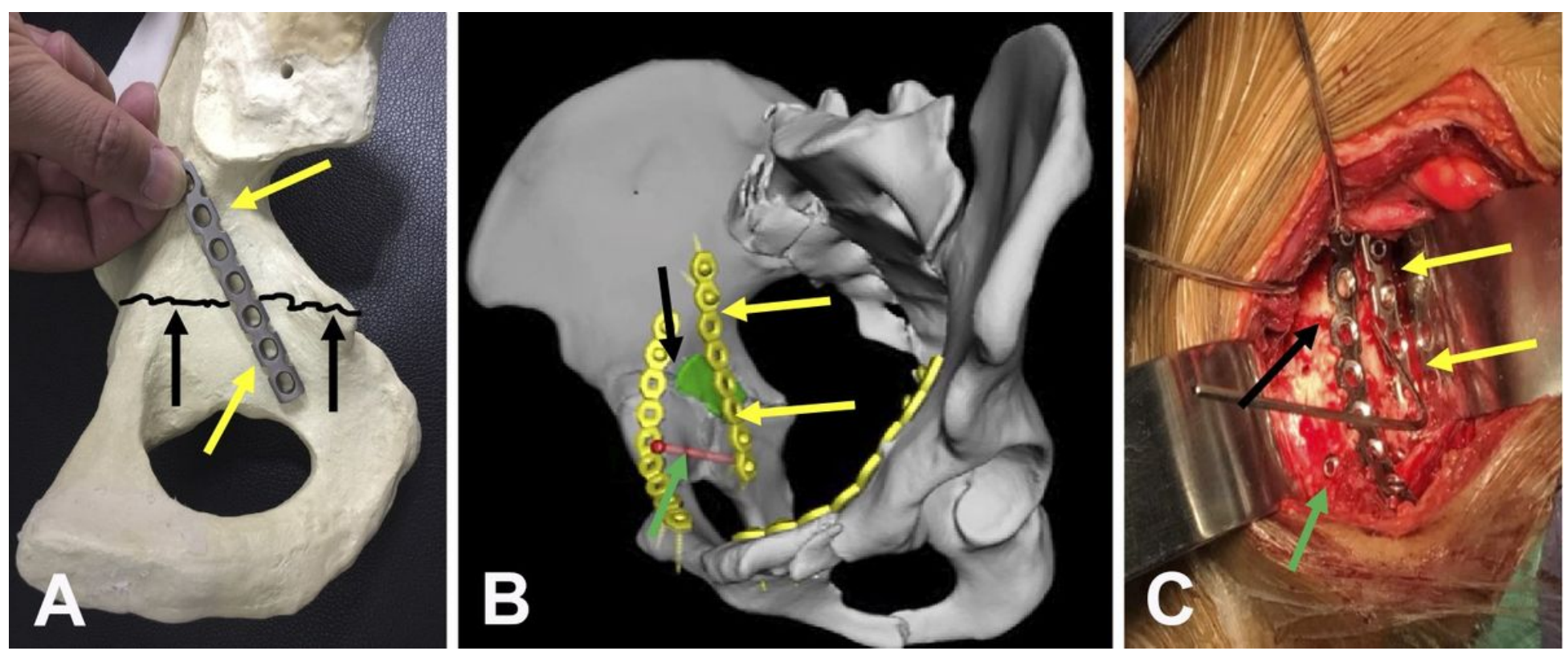

\section{Figure 1}

Illustrations of orientation of the ilioischial plate with (A) sawbone, (B) simulated patients, and (C) real patients. The ilioischial plate should be intraoperatively contoured over the pelvic brim to counteract posterior column medial displacement. Displaced quadrilateral surface fragments were meticulously buttressed with the bridging effect. The plate was then anchored with cortical screws to the iliac fossa 
and the safe zone between the sciatic buttress and the ischial spine. An additional lag screw can be inserted from anterior to posterior direction within the safe zone to provide further fixation of the posterior fragments. The black, yellow, and green arrows indicate the fracture line, ilioischial plate, and lag screw, respectively.
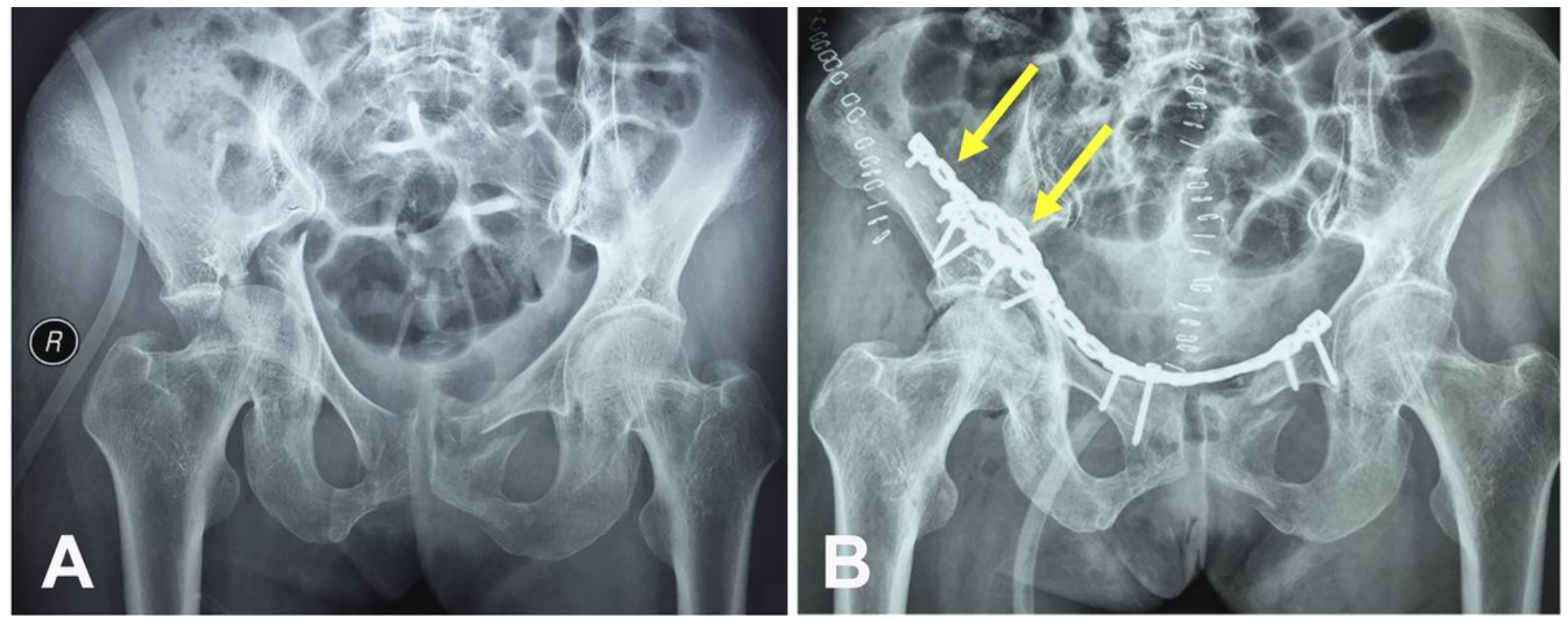

\section{Figure 2}

A 61-year-old female with a T-shaped (right-side) acetabular fracture. Anteroposterior radiographs showing (A) preoperative displacement of the fracture components and (B) postoperative fracture reduction and internal fixation with two anterior spring plates. The yellow arrows depict the ilioischial plate.
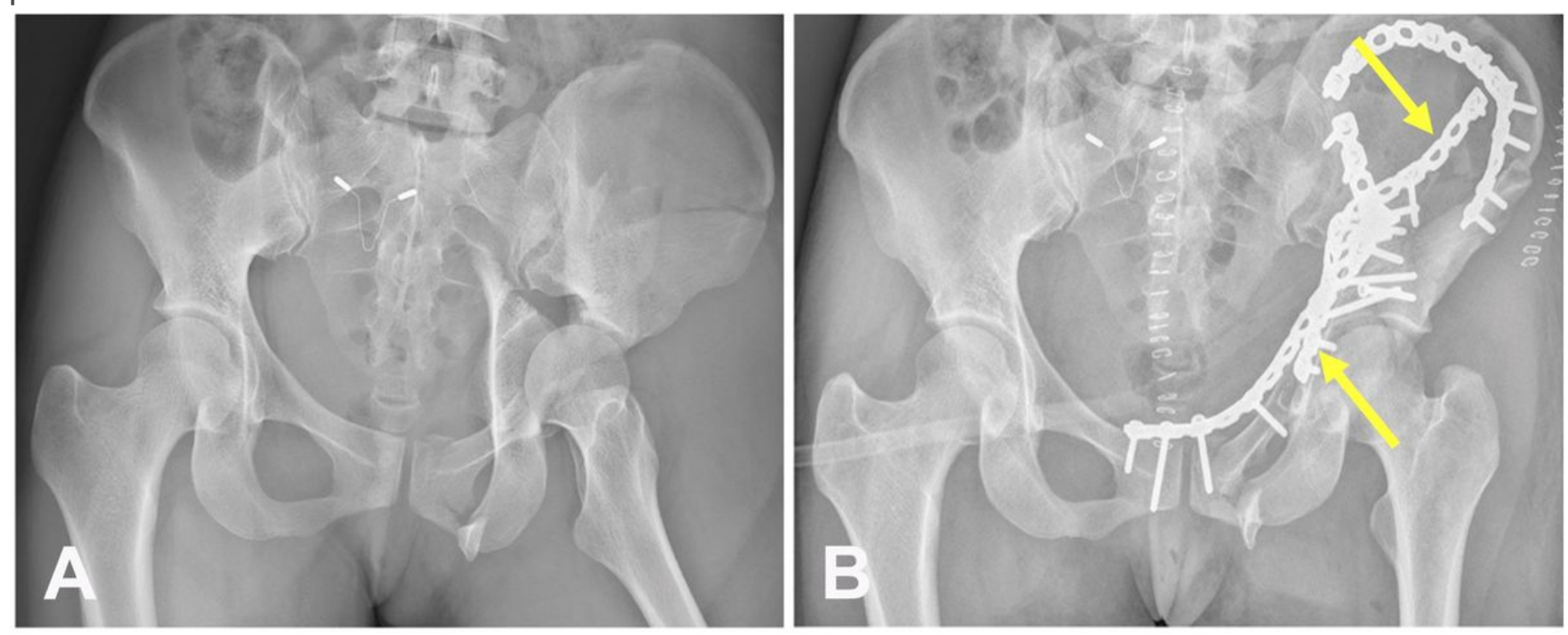

\section{Figure 3}

A 32-year-old female with an acetabular fracture (left-side) involving both anterior and posterior columns. Anteroposterior radiographs showing (A) preoperative displacement of the fracture components and (B) 
postoperative fracture reduction and internal fixation with four spring plates. The yellow arrows depict the ilioischial plate. 